\title{
Using Participatory Methodologies to Achieve Change in Valuing Volunteering
}

\author{
Elizabeth Hacker
}

\begin{abstract}
The global action research project Valuing Volunteering explores how and why volunteering contributes to poverty reduction and sustainable positive change, and the factors that prevent it doing so. The research modelled an approach that empowered local people to better understand the challenges they face, acquiring valuable learning for volunteering for development organisations to design initiatives better able to alleviate poverty. Volunteers were at the centre of the participatory action research processes established within each of the four Valuing Volunteering countries (Kenya, Mozambique, Nepal and the Philippines). This article explores how volunteers used participatory approaches to help communities achieve lasting social change and alleviate poverty. Examples from the four countries show the benefits of using participatory approaches, and the advantages of specific participatory tools, such as system mapping. Valuing Volunteering shows both what volunteers can achieve through using a participatory approach and participatory methods, and what can be learnt from the challenges faced.
\end{abstract}

\section{Introduction}

The global action research project Valuing Volunteering explores how and why volunteering contributes to poverty reduction and sustainable positive change, and the factors that prevent it doing so (Burns et al. 2015). Commissioned by Voluntary Service Overseas (VSO), the research was conducted over two years in 2012-14 in four countries: Kenya, Mozambique, Nepal and the Philippines. A lead researcher (an international volunteer researcher recruited by VSO) was deployed in each country to work with locally based partners and volunteers to develop case studies that used an action research approach to understand volunteering's impact. The 12 case studies cover a range of volunteer interventions, including VSO projects that utilised international volunteers, self-help and community volunteering initiatives and national volunteering schemes. The breadth of the study aimed to inform learning and practice for both VSO and the volunteering for development (VfD) sectors on how to effectively use volunteers to achieve sustainable change (see Burns and Howard, this IDS Bulletin).

The Valuing Volunteering research modelled an approach and way of working that empowered local people to better understand the challenges they face, and acquired valuable learning for volunteering for development organisations to design initiatives better able to alleviate poverty. A strong participatory research process was embedded in all four Valuing Volunteering countries. Two interrelated methodologies were used - participatory systemic inquiry (PSI) and systemic action research (SAR) - to facilitate many different types of participatory inquiry to find out what was going on and why (Burns 2007, 2014). Group discussions were then held to make sense of the data and generate frameworks for action where possible. Data collection methods included a range of participatory methods: for example focus groups, problem walls, informal discussions, digital storytelling, rivers of experience, network strings and system mapping.

Each inquiry brought together a diverse range of volunteers, local partners, community members and decision-makers to support their understanding of the development issues faced and the role of volunteers in addressing them (Burns et al. 2015). Inquiries explored different kinds of volunteer interventions and different development issues, such as education, health and the environment. The inquiries involved 3,794 participants across the four countries $(1,282$ in Kenya, 872 in Mozambique, 383 in Nepal and 
1,257 in the Philippines), making the process robust and ensuring a myriad of perspectives are reflected from a range of communities in the global South.

This article explores how volunteers used a participatory approach (PSI and SAR) to help communities achieve transformative social change and alleviate poverty. It explores the benefits of using participatory approaches (PSI and SAR), and the advantages of specific participatory tools, such as system mapping, that were utilised within the overarching research approach. Section 1.1 shows how volunteers can facilitate a participatory approach that develops local capacity to gather in-depth context-specific information, revealing issues from the bottom up. Section 1.2 shows how volunteers can be supported to reflect on the complexity of their contexts and understand how their volunteer efforts affect and are affected by this complexity. Section 1.3 explores how volunteers can co-generate ideas that take this complexity into account, building frameworks for action that are grounded in an understanding of local realities. And Section 1.4 shows that by putting these solutions to the test, and repeating iterative action research cycles, these solutions can be 'fine-tuned' so that they become more responsive to community needs and better able to achieve sustainable positive change. The challenges for volunteers and for VfD organisations in implementing a participatory action research approach and utilising participatory methods are also discussed.

\subsection{A systemic approach: engaging multiple stakeholders and using a variety of methods to see 'more of the whole'}

Valuing Volunteering used PSIs to support local communities to map a system of actors, actions and contexts as a baseline, from which change could be assessed against (Burns et al. 2015). Within each country there were between three and nine PSIs across varied research sites. PSI locations ranged in size from a school catchment area in a district of the far west of Nepal, the Korogocho informal settlement in Nairobi, to a barangay (smallest administrative division) on the Philippine island of Palawan. Each inquiry involved coalitions of volunteers, local partners, community members and decision-makers who were brought together and supported to nurture their understanding of the development challenges they faced and the role of volunteering in addressing them (Burns et al. 2015). Usually, at this initial stage, the Valuing Volunteering researcher worked with a small group of local and/or national volunteers and possibly a partner organisation who, once trained, worked with the researcher to use participatory methods to collect and gather information from a range of stakeholders in the research site.

The PSIs began with broad questions to deepen understandings of the context and the wider system dynamics. Where a volunteer intervention was already under way, the inquiry did not solely focus on the specific area of intervention. Instead, the inquiries went beyond the typical starting points identified for a project, going deeper to ask how people, processes and the environment that they were situated within influenced one another and the pathway to change (Burns et al. 2015). For example, in the far west of Nepal, although the inquiry was focused on education, the complex political, ethnic and caste dynamics that underlay many interactions within the focal communities were taken into account and later found to affect the success of the formal education intervention.

The inquiries engaged multiple stakeholders. This avoided a single starting point or perspective, and helped to build a nuanced picture of the context from different locations in the system (Burns 2007). Often the range of stakeholders included in the inquiry evolved as the inquiry did. For example, on the island of Palawan in the Philippines, one storyteller was asked to recommend the next. This snowballing technique utilised residents' local knowledge and ensured the inclusion of a broad range of participants. This avoided including only the 'usual suspects': for instance, in the Philippines and Nepal, inquiries which initially included only school-attending youth, were expanded so that the out-of-school children became participants in the process. Typically different groups were engaged with one by one; for example, school teachers, parents, students and out-of-school children, so that their perspectives could be fully explored.

In addition to the inquiries engaging a range of stakeholders, the participatory methods used to enable participants to observe and gather information were varied depending on the particular questions being asked and who they were being asked to. Care was taken to adopt methods which allowed participants to express themselves, feel engaged in the process and not feel compelled to give the 'right answers'. Visual and creative techniques allowed participants to express issues that some may have found difficult to express in words, perhaps because of the sensitive nature of the subject area or social norms and taboos. In the 


\section{Systems mapping}

Systems mapping can be used to understand the interrelations between issues and stakeholders and navigate mutually agreed ways forward. Systems mapping usually follows a PSI, where volunteers (often community based) are trained to gather information from multiple stakeholders. The data collected may include transcripts from interviews and focus groups, notes from informal conversations and observations, photographs, and pictures and maps drawn by community members to portray feelings, power relations or information that is difficult to portray verbally. Maps are then created, usually in teams of between three and ten volunteer researchers. The maps can be large - several metres in length and breadth - which allows for several researchers to work on them at the same time, and for them to hold a large amount of data. At the start of the process, a brainstorming session with the volunteer researchers that identifies the key emergent issues from the inquiry can help organise the data into initial themes. These key themes and issues are recorded onto the map, spaced logically so that related themes are clustered together. A standard colour coding is used in order to organise the data and allow comparisons across different maps. Key issues are written large in red pen. Beneath a key issue, verbatim quotations that expand and support the issue are added in black ballpoint pen, the stakeholders involved are written in blue, and factual information or observations (for example, 'women rarely spoke during the focus group discussion') are added in green.

Once the data have been transcribed, the relationships between issues and stakeholders are analysed as a group. Possible links or causal pathways between entries on the map are identified and lines drawn to indicate a relationship. Some maps revealed the power dynamics that affected issues - for example, in Mozambique, maps included the relationships of trust between volunteers and government officials. Barriers and opportunities for change, ideas for action and areas where more information is needed are also indicated.

This process often took place over one or two days. The maps became works in progress, and were added to as new information was collected and as thinking developed.

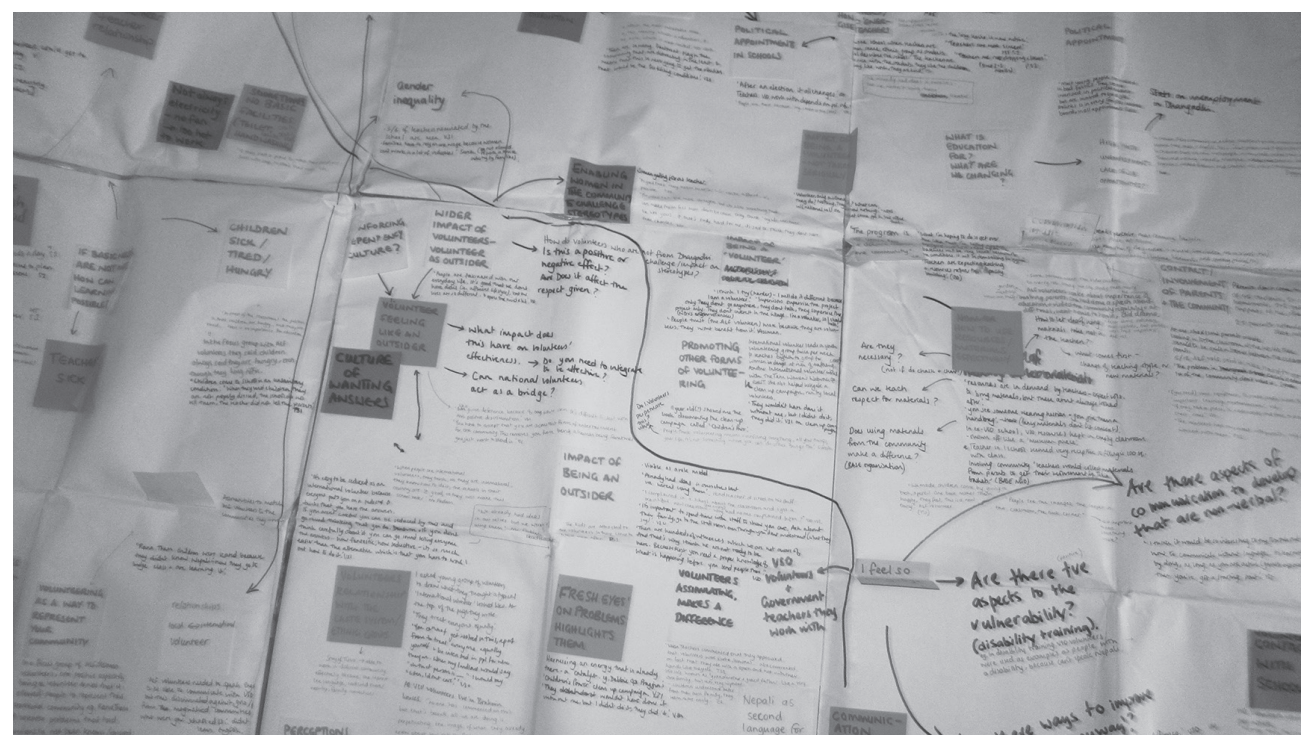

Above is a systems map constructed by members of a local research team in Nepal.

Philippines, visual techniques helped local residents understand what knowledge and attitudes had been internalised and to reflect openly on whether and why illegal fishing practices continued (Aked 2014). When residents were asked to draw the relationship between the environment and their livelihoods in the past, present and future, they drew pictures of illegal fishing activities, with some fishermen drawing a picture of their nets damaging coral which had happened the previous night. It could 
be difficult to elicit such open responses from more traditional research methods. In Nepal, visual and ethnographic methods were critically important to allow insights to emerge about the more hidden aspects of far western Nepalese society, such as the subtleties of caste, ethnic and gender relations. For example, living with Nepalese teachers for extended periods during field visits revealed the high domestic work-load of female schoolteachers and subtle forms of discrimination between castes and ethnic groups in the focal communities.

Informal 'street walking' sessions where time was spent in the community talking informally with residents, allowed for individuals and groups to be reached who may not have felt comfortable or able to engage in a more formal setting; for example, outof-reach children and their parents in Nepal, and those living with HIV/AIDS in Mozambique. In Kenya, Valuing Volunteering experimented with a storytelling approach which mobilised local 'scribes' to talk to people in their communities. Using short 'story forms', participants were asked to 'tell a story about a time when a person or an organisation tried to help or change something in your community'. Crucially, by letting the participants choose the topic with minimal prompting, they were empowered to talk about what was important and relevant in their communities. With no specific initial questions, the approach avoided a common pitfall of participants only talking about what they think the interviewer/ story collector wants to hear about (Lewis 2015). ${ }^{1}$

These PSIs provided volunteers with in-depth, context-sensitive insights within a relatively short time frame. The inquiries began broadly, with an overarching goal to understand where, when and how volunteering is effective, and with questions that went beyond project parameters. This breadth allowed interesting insights about the ecosystem of volunteering to emerge that would have been difficult to reach had the focus been on a specific intervention area. For example, in Kenya the PSI revealed that potential volunteers were dis-incentivised from volunteering with local community-based organisations $(\mathrm{CBOs})$ because these organisations were unable to compete with the stipends and allowances provided by large non-governmental organisations (NGOs). As a result, the community's local capacity to lead its own development was being eroded (Lewis 2015).

Ensuring a broad range of stakeholders are involved allows a bigger part of the system to be seen. Participatory methods, particularly those which allow volunteer researchers to make use of informal spaces, such as street walking and storytelling, help ensure that groups and individuals whose voice is often ignored by development organisations and governments, are included in building a picture of the system. Using local volunteers can be highly effective because they have access to these spaces and can be from a variety of backgrounds themselves, acting as a useful starting point to ensure a range of perspectives are collected.

\subsection{Providing the tools to understand complexity}

Inquiries progressed from enabling volunteers to gather information, to supporting them to reflect on the connections and interrelationships between issues in multi-stakeholder groups. In all four countries, systems maps were used to facilitate this process. Systems mapping is a technique used to better understand issues and relationships in complex environments by bringing together information and views from a number of participants onto one page (Aked 2013). Maps were created that included issues that were prominent in the inquiries.

The maps allowed volunteers to quickly see the issues and their interrelationships and how volunteer efforts interacted with the wider social, economic and environmental concerns of the communities involved. It enabled them to deconstruct and better understand the complex factors that caused poverty. For example, the VSO education intervention in Nepal mobilised volunteers to improve school enrolment and retention rates. Volunteers worked with schools to improve classroom learning, and with out-of-school children to bring them up to speed to enter mainstream education. However, mapping the system revealed that complex migratory patterns, insecure livelihoods and a lack of culture and understanding of formal education were powerful systemic drivers which kept the hardest to reach children out of school and involved in rural or domestic labour. The intervention had limited measures to address or accommodate these migration trends, issues around insecure livelihoods and the community's education culture (Hacker 2014).

On the island of Palawan in the Philippines, rich data about the drivers of illegal and unsustainable fishing practices were collected by local volunteers and local organisations mobilising volunteer educators. The data revealed that local volunteer action was not aligned to these drivers: local volunteer action focused on tree planting and coastal clean-ups, and volunteer educators raised awareness of the dangers of environmental degradation. 
The poor governance of fishing laws, low income levels and lack of alternative livelihoods that were driving the behaviour that badly affected the environment were not addressed by volunteers and the organisations mobilising them. The systems map enabled volunteers and volunteer organisations to see the complexity of the situation facing the fishermen, and to consider how to best deploy volunteers at the intersection between poverty, environmental and livelihood issues (Aked 2013).

Systems mapping also enabled participants to see how blockages in one part of the system could affect pathways of change, helping volunteers and VfD organisations to understand why an intervention hadn't had the impact expected. In Nepal, systems mapping was effective in supporting volunteers to understand the power dynamics that needed to be overcome in order to be effective. The mapping sessions revealed how strict hierarchies within schools which were overlaid by more subtle gender and caste hierarchies, affected the extent that learning from volunteers could be cascaded from the focus teacher (who worked closely with the volunteer) to other teachers within the school. These power dynamics made it more difficult for the intervention to gain momentum and for the whole school to improve teaching techniques. In Maputo, Mozambique, systems mapping also showed that relationships within the system could affect volunteer impact. Church-based volunteers realised that their 'outsider' status and lack of relationship with local institutions and governance officials limited their access to resources.

Importantly, because the maps were created by and presented to multiple stakeholder groups, they allowed for negotiation between different perspectives which could facilitate shared decisionmaking (Aked 2013). In Palawan, the Philippines, Aked found that 'once you have made visible the relationships between different issues, people can reach a consensus on the need for action even if their starting points are completely different'. For instance, during the systems mapping exercise the fishermen's concerns were about protecting their way of life, the women wanted to see progress and a role for themselves in it, and the treasurer of the volunteer group was motivated by the impact of reduced fishing stocks on family relationships, having suffered a family breakdown himself. As a tool, it helped to turn differences into shared decisions.

In Nepal, the systems mapping process revealed deep divisions and distrust based on ethnicity and caste in one focus inquiry school, particularly among the school governors, parent--teacher group and teaching staff, that was affecting a volunteer intervention. However, providing a neutral space for multiple stakeholders to analyse issues, and discuss the linkages and connections between them - returning to the maps as a focal point when necessary - was useful when discussing sensitive issues around ethnicity and caste. At the end of this session, the group decided to hold a meeting to improve relations between the different groups (Hacker 2014).

As well as enabling volunteers and communities to understand complexity, the process helped those living in poverty and the most marginalised to engage with relevant stakeholders to try to ensure the realities of their lives were understood. The maps became a tool for negotiating with other stakeholders in the system whose support was required to move to action. It allowed participants to tell a story of the wider system, and practically speaking, was an efficient way to communicate a high level of information and complexity: a single piece of paper that could be shared with lots of different stakeholder groups (Aked 2014). In Palawan, the fishermen were able to take the map to the City Agriculture Office to show the connections between the system's human dynamics (i.e. a lack of alternative livelihoods for fishermen) and continued illegal fishing and ecological degradation. Although the office were initially defensive, asserting that a myriad of livelihood projects had been instigated to assist fishermen, the map enabled them to unpack the causes for why these hadn't worked, highlighting the top-down nature of the interventions, which were agricultural rather than marine based, and misaligned with the interests and energies of local people.

\subsection{Co-generating ideas to solve complex problems}

Systemic maps allowed for multiple stakeholder groups to develop in-depth understandings of the local context. Volunteers and VfD organisations were then able to generate their own solutions and build frameworks for action that were grounded in an understanding of local realities. It allowed them to be more directed and precise in their approach because they began to understand where volunteer action was best placed, and how volunteer efforts could be affected by systemic factors such as the political and economic context.

Valuing Volunteering used a number of participatory techniques to support communities to explore the possibility of locally devised solutions and to begin 
developing the leadership qualities that volunteers needed in order to take these ideas forward. This could be difficult in situations where there was an established culture of looking to outsiders (e.g. political leaders, officials, NGOs) for guidance, and disillusionment in the long-term sustainability of development interventions. In Palawan, participatory tools were used to support local volunteers, fishermen and fishing community members, to devise solutions and explore their role in the change process. One exercise, 'Plugging the Leaks' (developed by the New Economics Foundation) used a bucket full of water, with the water used to represent community wellbeing. The group brainstormed what was needed in the community to make things go well, for example fish in the sea, investment in livelihoods, etc. The group then looked at what happens to a bucket with holes drilled into it when water is poured in: the water leaks out. The group brainstormed what the holes represented - leakages of resources (e.g. diseased crops, time lost in gossip, etc). Ideas about potential projects that could close the leaks were then discussed. The participants voted for the best three ideas to take forward, which included the development of a snorkelling site and abalone culture, and self-selected onto working groups depending on the idea that interested them most (Aked 2014).

In the far west of Nepal, volunteers worked with parents, teachers and local partners to devise solutions to issues that were affecting children's learning outcomes and ability to stay in school. During action planning sessions, the group decided to address both short-term issues and underlying drivers that required long-term solutions. Although international volunteer efforts were focused on building the capacity of teachers in child-centred learning, during the participatory inquiry and systems mapping, it emerged that this was difficult given the poor infrastructure of the early years education school buildings (e.g. holes in the roof meant that learning materials were damaged and impossible to store safely). The group decided to form an early years parent-teacher group which went on to work with a local partner to lobby local officials and secure funding for a new roof. Longer-term issues that went beyond the school boundaries were also identified during the system mapping meetings: one issue discussed was that parents who were illiterate seemed less likely to send their children to school. Parents and teachers felt that they could encourage parents to attend literacy classes as a long-term aim to improve the culture around valuing education in the community. Coincidentally, there were literacy classes beginning shortly after the meeting and several members of the group agreed to attend and encourage others to join them (Hacker 2014).

\subsection{Testing locally devised solutions in 'real time'}

Action research is based on the principle that we learn as much if not more from action (Burns 2007). Putting the devised solutions into action enabled volunteers and volunteer organisations to test and adapt the locally devised frameworks of action (or theories of change) in real time. In many cases, testing locally devised solutions highlighted unintended consequences or had unexpected results. But by repeating iterative cycles of action and analysis, it was possible to reflect on a particular action or approach, adapting it according to what was learnt. In this sense, the iterative cycles allowed volunteers and VfD organisations to 'fine tune' theories of change, so that solutions were more responsive, impact was targeted at where the need was greatest and actions took into account potential blockages and hurdles in the system. The SAR approach provides tools for volunteers to tackle these blockages, rather than be disillusioned by them. This is particularly important in contexts where there is a high level of cynicism regarding the success of community development projects, and can help to achieve sustained engagement and a feeling of ownership for local volunteers.

In Nepal, the first round of action research with the parent, teacher and volunteer group showed how action can lead to unintended consequences and require theories of change to be adapted. Having decided to try to improve adult literacy in their community, parents, teachers and other local stakeholders reconvened two months later to discuss progress. As mentioned above, literacy classes began in the area shortly after the first meeting and several members of the group agreed to attend and encourage others to join them. At the next meeting, while four of the participants had attended classes, they reflected that learning outcomes were mixed. The literacy teacher could not speak their ethnic group's language, and the participants found Nepali difficult to understand and learn without being instructed in their mother tongue. Furthermore, the parents reported that the freedoms of some of the women were restricted by their husbands and they were not allowed to leave the house to attend the classes. How their framework for action could be adapted was discussed in light of these insights. An international volunteer who attended the meeting and worked closely with the district education office agreed to speak with his colleagues about the need 
for literacy teachers who could speak languages other than Nepali. It was more difficult to tackle issues around gender relations in the community, particularly as this relates to long-established cultural norms, but had the action research process continued long term, this could have formed a further inquiry line.

In Mozambique, the process highlighted the nonlinearity of change and the fact that there are often contradictions and dilemmas associated with taking action. Church-based youth volunteers working to raise awareness to improve sexual health in their communities used participatory methods to understand their impact and the barriers to their effectiveness. Through a relationship mapping exercise, they found that a major barrier to them more effectively raising awareness in this area was their weak relationships with local governance structures and officials, in particular the local elected official, the secretario do bairro (SDB). This meant their access to funding and spaces was restricted and they had few places to meet and organise. Volunteers decided to take action on two fronts: firstly, to build capacity to take action within the local system of service delivery by developing their working relationship and linkages to individuals and institutions such as the SDB; and secondly, to conduct a locally based participatory inquiry to develop an in-depth picture of the issues faced by the community and to develop a plan of how to respond to these. The inquiry helped to ensure the volunteer responses were rooted in community research, but it also gave them legitimacy and standing when approaching the SDB. This brought gains: volunteers were invited to attend local governance meetings regularly and were provided with links to relevant local organisations (Picken 2014). ${ }^{2}$

But moving from the observation and reflection phase of the project to the action phase also raised issues and questions for the volunteers. Progress was made in building relationships with local governance structures, and the SDB showed a supportive attitude towards them which facilitated access to wider networks in the community. However, closer links with this political figure made them more susceptible to becoming politicised, as they were asked to link to party political youth groups. The volunteers felt that this risked how they could be perceived in the neighbourhood, highlighting a contradiction: on the one hand, building collaborative relationships with key political stakeholders provided endorsement and could allow barriers to be removed in terms of working with the community. On the other hand, these relationships risked compromising the volunteers' relative neutrality, making trusting relationships harder to build with community members.

The SAR approach, where the activistas were supported in reflecting on their findings, moving to action and then reflecting again on the actions taken, was important in showing that change is not linear. The process revealed that there are often contradictions and dilemmas associated with taking action. Using participatory methods allowed for the complexity of change processes to be observed and for emerging dilemmas to be reflected on and discussed collectively.

In the fishing village in Palawan, the Philippines, moving to the action phase led to important process learning, as a lack of local capacity to direct change was revealed. The leap between inquiry, reflection, planning and then finally the implementation stage can be difficult to manage. As Aked notes, 'there is not a direct link... between being able to see the potential levers for change and having the individual and organisational capacity to construct (and implement) a course of action that is responsive to local realities' (Aked 2014: 7). Despite local volunteers devising a framework for action based on the inquiry and reflection stage, the date of the meeting for residents to come together and implement actions came and went. Follow-up meetings revealed that the leaders of the working groups were unsure about what to discuss at the meeting. They didn't want to do it alone. This in itself was an important finding which was reflected on and actions planned to leverage more local level support to build capacity and mentor potential leaders. This involved recruiting a national volunteer with expertise in business, finance and livelihoods and exploring links with local government institutions and universities who could potentially support the volunteers' efforts.

The SAR process in the Philippines revealed the need for spaces which allow volunteers to develop their capacity to organise, meaningfully engage with different stakeholders and take action. In the Philippines, building local volunteers' capacity became a specific inquiry line and part of the SAR process itself. Even if not a specific inquiry line, engagement in the SAR process and gaining experience of using participatory tools and methods can have valuable side effects for volunteers as their capacity to challenge power dynamics increases. For example, in Mozambique, the inquiry process itself provided volunteers with the legitimacy required 
to participate and be heard in formal spaces where they would not usually have the opportunity to do so (whether due to overt or subtle hierarchies of power). In Nepal, a participatory digital storytelling workshop enabled early years teachers and community education volunteers from marginalised communities to reflect on their experiences of volunteering, but also helped to give them a voice when meeting with decision-makers. During the workshop seven short films were produced which highlighted issues faced by volunteers and the schools they worked in. The workshop gave a space for reflection, but also led to the start of action because they gave the volunteers an opportunity for these stories to be heard more widely and for action on a different scale. For example, by showing the stories at a meeting attended by government officials, NGOs and international non-governmental organisations (INGOs), their experiences and issues could be shared and their voice given strength. It was difficult for the participants to be involved directly in the discussion but the stories normalised their presence in a formal space and gave some participants the confidence to approach government officials at the end of the meeting to request that they check on the provision of resources to their school (Hacker 2014).

\section{Analysis}

The action research cycles and participatory methods used by Valuing Volunteering did allow community volunteers to begin to address some of the identified challenges that had previously affected the success of their efforts. It enabled some volunteers to be more directed and responsive in their approach and devise more nuanced theories of change that could continue to be adapted as their frameworks for action were put to the test. Despite these gains, the action research inquiries facilitated by Valuing Volunteering highlighted a number of major challenges for volunteers and VfD organisations using participatory tools and an SAR approach in high poverty contexts.

In all countries, researchers reported difficulties in sustaining volunteers' momentum throughout the duration of the process, which usually lasted between 18 months and two years. Maintaining the enthusiasm and interest of volunteers for a two-year period can be difficult, particularly if there is little or no financial incentive. In Korogocho, Kenya, the continually changing circumstances of the local researchers did present a challenge to the research in terms of maintaining the team's dynamics, as volunteers changed jobs, completed studies or secured new opportunities. In Maputo, Mozambique, a decrease in energy and interest of church-based volunteers was noted by the end of the 18-month process. For the fishermen in the Philippines, time away from fishing meant that their families would have to go with less, making it difficult for them to have the confidence to prioritise meetings (with uncertain ends) over other commitments.

Working in contexts where development projects usually offer more immediate gains (e.g. a training or financial resource) can exacerbate this. In Korogocho, time had to be taken to find volunteers who genuinely wanted to participate in the process rather than participate to receive a 'sitting allowance', a practice that had become commonplace amongst local NGOs and CBOs, eager to show a high number of volunteers on record. Time was invested in finding the 'right kind' of volunteer who was committed to alleviating poverty and open to learn. In addition, ensuring ongoing training was provided that allowed the local volunteers to steer the process as it evolved helped sustain engagement at each stage (Lewis 2015). In the Philippines, participants who were used to receiving tangible 'rewards' from development organisations (i.e. training or monetary resource) initially asked what they would receive in exchange for attending the Valuing Volunteering meetings. As Aked notes, in such contexts, the trajectory of the process may be slowed down because expectations and ways of working need to be redefined.

Supporting communities to generate ideas and design projects is often a much lengthier process than a more top-down approach that uses experts to push forward with ideas (Aked 2014).

Furthermore, the process can be lengthy. Lewis notes that in Korogocho the first stage of the process, the PSI, took much longer than first anticipated. Time was invested in building an in-depth understanding of the complexity of the local context and it was felt that undertaking activities before the full picture was better understood ran the risk of causing more harm than good. This along with disruptions caused by the Kenyan national elections, made organising other activities, such as establishing the research group, very challenging. As a result the research team did not properly start its work until May 2013, eight months after the start of the project (Lewis 2015).

When working with community volunteers, momentum can also be affected by the capacity held locally to move the process forward. While action research is democratic in nature, with one of its key underlying principles being that 'we can all do it', Valuing Volunteering revealed that cooperation 
between facilitators and participants in the action research process may need to be much more hands on in high poverty contexts (Burns 2007; Aked 2014). This didn't mean that researchers/facilitators reverted to leading or directing participants and volunteers. However, more time was required to build local capacity on how to, for example, organise and facilitate discussions and meetings. Without this, time could be lost in the six weeks or so between visits from the facilitator. In Palawan, it was felt that a consistent presence was required by a facilitator in the community in order to create momentum for change, particularly given the low capacity of local volunteers and the reassurances they required to give up valuable time to a process which was evolving and had uncertain outcomes.

Practical and logistical difficulties are also worth noting because of their impact on sustaining the momentum of the processes. These were wideranging: security concerns prevented the facilitator from operating in Korogocho; the complex linguistic terrain of the far west of Nepal made it difficult for some community members to participate in their mother tongue, with few translators available who could bridge the gap between English, Nepali and indigenous languages; in all countries follow-up meetings were missed due to facilitators being affected by bad weather in remote areas, political disruption and security emergencies. Such challenges largely present themselves because of the use of volunteers and facilitators from 'outside' the research communities, i.e. the international Valuing Volunteering research volunteers.

Using combinations of volunteers (i.e. international, national, local) to carry out PSI and SAR processes does present difficulties: in addition to the logistical challenges described above, researchers noted widely held expectations that as an international volunteer 'expert' they would provide funding, leadership and expertise. As one participant said in an SAR workshop in Korogocho, 'they see a mzungu [white person], like in this meeting, and they think there is money' (Lewis 2015: 95). Roles needed to be carefully negotiated and good facilitation skills were needed to ensure that researchers were seen as supporters rather than leaders of the process. Working in teams that combined different types of volunteer also had real advantages. For example, in Korogocho, although the international volunteer researcher was unable to work directly in this 'closed community', he was able to use his status as an outsider to mobilise volunteers to be involved (a local volunteer probably would have struggled to get this kind of buy-in) (Lewis 2015). These local volunteers then provided a bridge into the community, putting in over 1,000 hours of fieldwork, to build an in-depth picture of local realities in one of Kenya's biggest informal settlements. International volunteers can have catalytic effects when working with and motivating local volunteers.

Aspects of volunteering, for example, the length of time spent with communities and volunteers' embeddedness within these communities, can lend themselves to establishing strong action research processes. For example, in Kenya, the international volunteer could spend time working with local volunteers to identify and train participants to be involved in the process. This allowed for committed local researchers to be found who drove the process forward and created momentum. It was the local volunteers' embeddedness in their communities that allowed for in-depth insights to emerge from the participatory inquiries (Lewis 2015). In the Philippines, using an international volunteer meant that time could be spent building the capacity of local volunteers once this had been identified as a need. The continuity of the volunteers' presence allowed for the process to change course slightly to address this need, rather than solely focus on developing the more tangible livelihood outcomes. As a result, local people could have more ownership over the process as they were provided with tools to discover their own agency, rather than efforts to find and implement solutions being handed over to 'experts' (Aked 2014).

Having a continued presence also allowed volunteers to invest in building relationships with participants - a critical element in building a robust action research process, particularly when there aren't tangible and immediate outcomes or financial rewards for taking part. Living and working alongside co-researchers and participants as many of the volunteer facilitators did, meant there was a shared investment in the project. This helped to build trust between the volunteers, creating a sense that the volunteers (whether international, national or community based) were 'in it together'. This could help sustain the process, even when outcomes were uncertain, and was often an important step in giving communities the capacity to facilitate change for themselves from the bottom up.

There were many examples where Valuing Volunteering was able to facilitate a process that mobilised local volunteers to devise solutions to local problems. What was more difficult was to find the resources and establish firm linkages with local 
partners, for example development organisations or government offices that enabled actions to be effectively carried out. Often the tools and methods highlighted major systemic drivers (for example, a lack of livelihoods, poor governance) that were preventing change. These drivers often related to complex economic and social issues, and structural inequalities that resulted in certain groups having limited access to resources. But mobilising human resources alone is often insufficient for tackling such issues. For example, in the Philippines, system mapping revealed the need for the development of alternative livelihoods for fisherman for behaviour around illegal fishing practices to change. But there were no finances available for these alternatives to be fully explored and the fisherman had no financial incentive to transition to more sustainable fishing practices. There were no viable alternatives in place, and the time frame of the Valuing Volunteering project allowed limited time for proposal writing and fundraising to help secure funding that could develop any of these alternatives. Links with local organisations could be facilitated but Valuing Volunteering found the policies, protocols and financial requirements required to support citizens in their efforts to overcome poverty highly restrictive and unworkable within the project time frame (Aked 2014).

The availability of local resources and expertise was also an issue in Nepal. System mapping exercises highlighted issues that required long-term solutions, such as the effects of alcoholism on the social fabric of communities (which in turn was affecting school attendance), and low adult literacy. Such issues required long-term engagement, the capacity building of community members to tackle them, and the expertise of relevant professionals (e.g. health workers) who were not available. It could take years to realise impact in these areas, making sustaining momentum difficult given the two-year time frame of the project and high workloads of agricultural workers.

The research also highlights the risks of working with partners who do not share the same commitment to participatory working or are limited in their ability to embrace such an approach because of a need to meet existing project design, evaluation and budgetary requirements. In Palawan, the Philippines, Valuing Volunteering approached a national university during the first action research cycle, inviting them to support the community initiative to develop a snorkelling site (Aked 2014). The university shared Valuing Volunteering's commitment to livelihood development and environmental sustainability. Despite the apparent value fit with the university, the collaboration revealed the challenges of bringing on board partners who, in practice, have different ways of working. For example, a reef assessment conducted by the university failed to engage resident volunteers because they were not fully included in the process and were unable to see the reef or learn about conducting such assessments.

Volunteers play an important role in fostering the human resource required to devise and instigate change processes. But these findings suggest that using such participatory approaches would be more effective if such an approach to volunteering is built into existing development strategies so that the creative approach and social mobilisation potential of volunteers is complemented by better access to funding streams, resources and expertise. This is not without its own challenges. In Nepal, where a participatory action research process overlapped with an existing VSO education programme, important questions were raised about how such a process 'fits' in terms of programming. There were certainly challenges from 'layering change on change' - with the action research process being a disruption or intervention, sitting atop an existing intervention (Burns 2007). Often development organisations working with volunteers have to operate within fairly narrow parameters, and have predefined project budgets and monitoring and evaluation requirements which limit the adaptive capacity of interventions.

\section{Conclusion}

Volunteers were at the centre of the participatory action research processes established within each of the four Valuing Volunteering countries. The Valuing Volunteering case studies in Kenya, Mozambique, Nepal and the Philippines show both what volunteers can achieve through using a participatory approach and participatory methods, and what can be learnt from the challenges faced.

In Kenya, the local volunteer researchers recruited and trained by Valuing Volunteering to steer the research process in the informal settlement of Korogocho, Nairobi, show how local people can develop their critical thinking around development challenges and devise locally rooted solutions, in areas where top-down development interventions have helped to create a culture of dependency and scepticism around volunteering. In Maputo, Mozambique, church-based youth volunteers working to raise awareness on sexual health issues used SAR to renegotiate their position in the volunteer ecosystem and establish better links with formal government institutions and local 
partners. In the far west of Nepal, an SAR inquiry established alongside a VSO education project helped volunteers and community members understand the responsiveness of the programme to community needs and the underlying blockages that were affecting the intervention's success. And in Palawan, the Philippines, PSI and SAR were used to understand the impact of NGO-mobilised volunteer educators' awareness-raising activities on illegal and unsustainable fishing practices, and to empower local fishing communities to address the economic and livelihood issues that were driving this behaviour.

There are challenges for volunteers and for VfD organisations in implementing a participatory research approach and utilising participatory

\section{Notes}

1 For a copy of this case study, please contact Katie Turner at katie.turner@vsoint.org.

\section{References}

Aked, J. (2014) Riding the Waves of Change: The Challenges of Volunteering in Highly Complex Poverty Contexts; Reporting on Action Research Among Volunteers in Mangingisda, Palawan, Pasig City, Philippines: VSO Aked, J. (2013) When There Are No Fish Left in the Sea: Does Environmental Awareness Translate into Positive Action? Reporting on the Impact of Volunteering for Environmental Education in Coastal Communities of Palawan, Pasig City, Philippines: VSO

Burns, D. (2007) Systemic Action Research: A Strategy for Whole System Change, Bristol: Policy Press

Burns, D.; Picken, A.; Hacker, E.; Aked, J.; Turner, K.; Lewis, S. and Lopez Franco, E. (2015) The Role of Volunteering in Sustainable Development, Brighton and London: IDS and VSO methods, for example: establishing and keeping momentum of an action research process when working with volunteers who receive little or no financial compensation; local volunteers' and partners' limited literacy in change processes and participatory ways of working; and finding ways to negotiate a fit between these experimental ways of working and VfD's sometimes rigid monitoring and evaluation systems, project budgets and time frames. The Valuing Volunteering case studies provide important lessons for the VfD sector on how to use the potential of volunteers to mobilise and engage communities in participatory processes, while providing the financial and organisational support required for them to lead positive social change from the bottom up.

2 For a copy of this case study, please contact Katie Turner at katie.turner@vsoint.org.

Hacker, E. (2014) Education and Volunteering in Nepal: A Case Study from the Hill and Terai Regions, www.vso.org.uk/about/what-we-do/ volunteering-for-development/valuingvolunteering (accessed 22 June 2015)

Lewis, S. (2015) Community Volunteering in Korogocho, www.vso.org.uk/about/what-wedo/volunteering-for-development/valuingvolunteering (accessed 6 July 2015)

Picken, S. (2014) Community Youth Church Volunteers: Trust and Motivations; A Systemic Action Research Inquiry, www.vso.org.uk/about/what-we-do/ volunteering-for-development/valuingvolunteering (accessed 6 July 2015) 